\title{
A theoretical study of thorium titanium-based alloys
}

\author{
K. O. Obodo ${ }^{1 *}$ and N. Chetty ${ }^{1,2 \dagger}$ \\ ${ }^{1}$ Physics Department, University of Pretoria, Pretoria 0002, \\ South Africa; ${ }^{2}$ National Institute for Theoretical Physics, Johannesburg, 2000, South Africa
}

\begin{abstract}
Using theoretical quantum chemical methods, we investigate the dearth of ordered alloys involving thorium and titanium. Whereas both these elements are known to alloy very readily with various other elements, for example with oxygen, current experimental data suggests that Th and Ti do not alloy very readily with each other. In this work, we consider a variety of ordered alloys at varying stoichiometries involving these elements within the framework of density functional theory using the generalized gradient approximation for the exchange and correlation functional. By probing the energetics, electronic, phonon and elastic properties of these systems, we confirm the scarcity of ordered alloys involving $\mathrm{Th}$ and $\mathrm{Ti}$, since for a variety of reasons many of the systems that we considered were found to be unfavorable. However, our investigations resulted in one plausible ordered structure: We propose $\mathrm{ThTi}_{3}$ in the $\mathrm{Cr}_{3} \mathrm{Si}$ structure as a metastable ordered alloy.
\end{abstract}




\section{INTRODUCTION}

Thorium, a heavy metal, combined with various elements such as boron, carbon, nitrogen and oxygen has been studied both theoretically ${ }^{1-3}$ and experimentally ${ }^{4-6}$ in relation ${ }^{2}$ to a range of physical, chemical, electronic, and thermophysical properties, often exhibiting high density, good thermal conductivity and good mechanical properties. The applications of Th-based alloys are wide-ranging. These include uses in nuclear reactors and in the aerospace industry because of its good corrosion resistant properties and high melting points. Th binary systems exist in various ordered alloys such thorium monocarbide (ThC), ${ }^{7}$ mononitride $(\mathrm{ThN})^{8}$ and monoxide ThO ${ }^{9}$ ThO crystallizes in the rock salt structure, while $\mathrm{ThO}_{2}$ crystallizes in the fluorite structure. $\mathrm{ThB}_{4}{ }^{10}$ and $\mathrm{ThB}_{6}$ exist in the $\mathrm{ThB}_{4}$ and $\mathrm{CaB}_{6}$ structures.

Titanium, a relatively light metal is strong, has excellent corrosion resistant properties and a high melting point. Given its relatively small atomic size, Ti can be easily impregnated into materials without radically altering the host crystal structure. Therefore, $\mathrm{Ti}$ is a versatile element for alloying and modifying the properties - especially the strength properties - of materials. Applications range from additives in paints to metals for aerospace and industry. In this way, Ti is useful for engineering the properties of materials. Ti-based oxides are very well known, for example $\mathrm{TiO}_{2}$ exists in the equilibrium rutile, and the metastable anatase and brookite structures, and the high pressure monclinic and orthorhombic forms. Ti also combines very readily with other metals, such as Pt in a variety of crystallographic forms and stoichiometries.

It would seem, then, that ordered alloys involving Th and Ti should form readily when in fact this is not the case. Experimental evidence by Carlson et al. ${ }^{11}$ dating back to 1956 still appears to be the only authoritative view on this subject, although similar results were found by Pedersen $e t$ al. in $1980 .{ }^{12}$ Carlson et al. discovered that Th-Ti forms a simple eutectic at $1190^{\circ} \mathrm{C}$ and at 12 wt $\% \mathrm{Ti}$, with no intermediate phases. Pedersen et al. found the eutectic composition to be 13.26 wt $\%$ Ti. The solid phases at the eutectic are $\alpha$-Th (cubic) and $\beta$-Ti (hexagonal). Carlson et al. concluded that solid solubilities at room temperatures are negligible. They argued that since the atomic radii of Th and Ti differ by about $22 \%$, and because the Hume-Rothery rules for alloying suggest that extensive solid solubility should not occur between metals differing in size by more than $15 \%$, the dearth of compounds (ordered or not) should not be surprising. Furthermore, they argued that since there is a marginal difference in the electronegativity of these two elements (the electronegativities are 1.3 for $\mathrm{Th}$ and 1.5 for $\mathrm{Ti}$ ), compound phases should not form readily. 
Since the authoritative experimental work done on these systems is more than fifty years old, and given the technological interest in both $\mathrm{Th}$ and $\mathrm{Ti}$, and the potential scientific and technological interest in alloys involving these elements, we have investigated these alloy systems using modern theoretical quantum chemical methods, which over the past two decades has proven to be an excellent tool to study such systems for a variety of material properties.

In the next section we present a brief description of our theoretical and computational methodology. In Section III, we present analysis on various ordered alloys of Th-Ti based systems with the aim of investigating their stability. In Section IV, an in-depth analysis of the bonding and electronic character of our proposed stable alloy is presented. Finally, we present our conclusions in Section V.

\section{METHODOLOGY}

All calculations were performed using density functional theory ${ }^{13}$ as implemented in the VASP code. ${ }^{14}$ We used the $\mathrm{PBE}^{15}$ and PBEsol exchange-correlation functional ${ }^{16}$ for the generalized gradient approximation (GGA). A kinetic energy cutoff of $500 \mathrm{eV}$ was chosen to ensure adequately converged total energies for the alloy systems under consideration. A Monkhorst-Pack ${ }^{17}$ grid of $12 \times 12 \times 12$ was used to sample the Brillouin zone (BZ) and Methfessel-Paxton smearing ${ }^{18}$ with a width of $0.2 \mathrm{eV}$ was used to integrate the bands to the Fermi level. The total energy, electronic band structure and density of states (DOS) were calculated using the tetrahedron integration method with Blöchl corrections. ${ }^{19}$

We tested our calculations with so-called Hubbard-U corrections of $1 \mathrm{eV}$ for the onsite correlation energy, which for Th could in principle be important since it is an actinide. Our investigations showed that the differences in a range of results involving the physical structure, the electronic structure and the energetics over the conventional GGA approach were negligible. This, in hindsight, is not surprising since Th has a closed $f$-shell of electrons with no highly localized orbital. All results subsequently presented have not included the Hubbard correction.

A method using the least-squares fit, ${ }^{20}$ as implemented in the MedeA-MT module, was used to obtain the elastic constants. This method uses the tetrahedron method for the Brillouin zone integrations. The elastic properties are calculated from the Hill value which is a geometric mean of the Voigt and Reuss values. The eigenvalues of the stiffness matrix gives an indication of the mechanical stability of the systems under consideration. The Hill values were used in the 
estimation of longitudinal, shear and mean sound velocities, and the Debye temperatures. ${ }^{21}$ The phonon dispersion curves and lattice dynamics were determined using linear response theory as implemented in the MedeA-PHONON package.

The heat of formation for each system was calculated by taking the difference between the total energy of the alloy unit cell and the sum of the energies of its constituents in their respective bulk elemental forms. The heat of formation of the system is strongly dependent on the spin-orbit coupling (SOC) due to the presence of relativistic effects in this heavy atomic systems.

Lu et al. ${ }^{22}$ used the same method based on the elastic, valence charge density distribution and phonon dynamics to study ThN, which has been experimentally synthesized with an experimental lattice constant of $5.154 \AA$ and bulk modulus of $175 \mathrm{GPa}$. This validates the approach used in this study. Therefore, we applied our methods to studies of pristine cubic Th and hexagonal Ti, and alloys of these elements in the $\mathrm{Cr}_{3} \mathrm{Si}, \mathrm{Cu}_{3} \mathrm{Au}, \mathrm{Al}_{3} \mathrm{Ti}, \mathrm{CsCl}, \mathrm{CuAu}, \mathrm{CuPt}$, and $\mathrm{NiAs}$ crystal forms. For the off-50:50 compounds we considered both the alloy and its dual alloy, e.g. both $\mathrm{Th}_{3} \mathrm{Ti}$ and $\mathrm{ThTi}_{3}$ in the $\mathrm{Cr}_{3} \mathrm{Si}$ structure.

\section{INVESTIGATION OF STABILITY}

The calculated elastic properties, phonon dispersion and heat of formation for thorium-titanium alloys is used to determine the elastically, dynamically and energetically stable alloys of $\mathrm{Th}-\mathrm{Ti}$ based systems.

The GGA (PBEsol) exchange correlation functional is an improved functional that takes into account the interactions present in closely packed structures. Hence, it is largely used in this investigation to understand the properties of $\mathrm{Th}$, Ti and Th-Ti based alloys. The results obtained using the GGA (PBEsol and PBE) for Th and Ti shows that the $\mathrm{PBE}^{23}$ exchange-correlation functional gives a slightly improved description of the lattice constants for Th and Ti. But, there is no fundamental difference in the description of the electronic properties of Th-Ti based alloys except in the heat of formation were the PBEsol gives consistently lower heat of formation compared to the PBE functional. Hence the results presented for the elastic properties, the electronic properties and the phonon dispersion considers the non-magnetic (NM) structure of Th-Ti based alloys using PBEsol. However, the heat of formation is strongly dependent on the exchange correlation functional along with the presence of relativistic effects. Therefore, these issues are taken into account only for the proposed stable alloy. 
The calculated lattice parameter using GGA (PBEsol) with the NM structure for Th (face centered cubic structure) is $4.95 \AA$, and Ti (hexagonal close pack structure) are $a$ of $2.90 \AA$ and $c$ of $4.59 \AA$. The inclusion of SOC leads to a slight change in the lattice parameters of these systems. The calculated equilibrium lattice constants $\left(a_{0}\right)$ for Th, Ti and Th-Ti based alloys is given in Table I for the NM cases. The lattice constants for Th-Ti based alloys in the NM phase shows there is an increase in the lattice constant with increase in Th concentration. This is expected because the Th atom is larger compared to Ti atom.

TABLE I. Lattice constant $a_{0}(\AA), \mathrm{c}(\AA)$ and heat of formation $(\mathrm{eV})$ of Th-Ti based alloys in various crystal structure using PBEsol in the non-magnetic phase

\begin{tabular}{rccc}
\hline \hline & $a_{0}(\AA)$ & $c(\AA)$ & $E_{f}(\mathrm{eV})$ \\
\hline $\mathrm{Th}$ & 4.95 & 4.95 & - \\
$\operatorname{ThTi}_{3}\left(\mathrm{Cr}_{3} \mathrm{Si}\right)$ & 5.32 & 5.32 & 0.740 \\
$\operatorname{ThTi}_{3}\left(\mathrm{Cu}_{3} \mathrm{Au}\right)$ & 4.28 & 4.28 & 0.683 \\
$\operatorname{ThTi}_{3}\left(\mathrm{Al}_{3} \mathrm{Ti}\right)$ & 4.11 & 9.19 & 0.685 \\
$\operatorname{ThTi}_{(\mathrm{CsCl})}$ & 3.60 & 3.60 & 0.433 \\
$\operatorname{ThTi}_{(\mathrm{CuAu})}$ & 3.21 & 4.62 & 0.585 \\
$\operatorname{ThTi}(\mathrm{CuPt})$ & 3.25 & 15.54 & 0.773 \\
$\operatorname{ThTi}_{(\mathrm{NiAs})}$ & 4.40 & 6.29 & 4.196 \\
$\operatorname{Th}_{3} \mathrm{Ti}\left(\mathrm{Cr}{ }_{3} \mathrm{Si}\right)$ & 6.05 & 6.05 & 2.514 \\
$\operatorname{Th}_{3} \mathrm{Ti}(\mathrm{Cu} 3 \mathrm{Au})$ & 4.78 & 4.78 & 0.930 \\
$\operatorname{Th}_{3} \mathrm{Ti}\left(\mathrm{Al}{ }_{3} \mathrm{Ti}\right)$ & 4.60 & 10.27 & 0.336 \\
$\mathrm{Ti}$ & 2.90 & 4.59 & - \\
\hline \hline
\end{tabular}

The possibility of a magnetic ground state in Th-Ti based alloys is examined. For this purpose, the spin-polarized (SP) calculations, assuming an initial ferromagnetic ground state are carried out for the Th-Ti based alloys. We found that the ground state of all the Th-Ti based alloys considered is NM, without any localized atomic magnetic moments. The NM and SP calculations yield similar lattice parameters and lead to NM ground state structures for both PBE and PBEsol functionals. Subsequently, the inclusion of relativistic effects leads to a slight (insignificant) change in the lattice constant and a (significant) change in the heat of formation compared to the NM and SP cases for both GGA (PBE and PBEsol) functionals. 


\section{A. Elastic properties}

The elastic properties of various ordered alloys of Th-Ti based systems are calculated for the NM phase. The elastic constants $\left(c_{i j}\right)$ obtained are shown in Table II. The criteria for elastic stability are: positive elastic constants, positive bulk, shear and Young's moduli, positive shear velocity (obtained from the shear sound velocity), positive longitudinal velocity (obtained from the longitudinal sound velocity). ${ }^{24}$ The systems that satisfy these criteria are elastically stable. However, this does not imply that such a system is dynamically stable. The negative elastic constant values for Th-Ti based alloy (in CuPt and NiAs structures) shows that these structures might be unstable because stability is related to sum rules.

TABLE II. Elastic constants $C_{i j}$ 's (values in GPa)

\begin{tabular}{rccccccc}
\hline \hline & $C_{11}$ & $C_{12}$ & $C_{13}$ & $C_{14}$ & $C_{33}$ & $C_{44}$ & $C_{66}$ \\
\hline $\mathrm{Th}$ & 69.55 & 52.54 & 52.24 & - & 69.55 & 49.32 & 49.32 \\
$\operatorname{ThTi}_{3}(\mathrm{Cr} 3 \mathrm{Si})$ & 196.1 & 53.39 & 53.39 & - & 196.1 & 60.55 & 60.55 \\
$\operatorname{ThTi}_{3}\left(\mathrm{Cu}_{3} \mathrm{Au}\right)$ & 122.2 & 77.94 & 77.94 & - & 122.2 & 39.64 & 39.64 \\
$\operatorname{ThTi}_{3}\left(\mathrm{Al}_{3} \mathrm{Ti}\right)$ & 85.33 & 114.2 & 68.40 & - & 135.6 & 40.48 & 61.26 \\
$\operatorname{ThTi}_{(\mathrm{CsCl})}$ & 105.0 & 67.23 & 67.23 & - & 105.0 & 9.020 & 9.020 \\
$\operatorname{ThTi}_{(\mathrm{CuAu})}$ & 146.1 & 22.40 & 65.65 & - & 97.75 & 17.26 & 26.05 \\
$\operatorname{ThTi}(\mathrm{CuPt})$ & 135.9 & 42.39 & 57.15 & -26.35 & 84.62 & -4.750 & - \\
$\operatorname{ThTi}(\mathrm{NiAs})$ & -1078 & 17.28 & -396.8 & - & 157.4 & - & - \\
$\operatorname{Th}_{3} \mathrm{Ti}(\mathrm{Cr} 3 \mathrm{Si})$ & 126.1 & 36.21 & 36.21 & - & 126.1 & 22.15 & - \\
$\operatorname{Th}_{3} \mathrm{Ti}\left(\mathrm{Cu}{ }_{3} \mathrm{Au}\right)$ & 91.85 & 58.40 & 58.40 & - & 91.85 & 45.24 & 45.24 \\
$\operatorname{Th}_{3} \mathrm{Ti}\left(\mathrm{Al} l_{3} \mathrm{Ti}\right)$ & 87.21 & 59.08 & 47.46 & - & 101.2 & 36.83 & 59.35 \\
$\mathrm{Ti}$ & 198.1 & 69.39 & 82.04 & - & 204.3 & 38.22 & 38.22 \\
\hline \hline
\end{tabular}

In Table III, we present the calculated bulk modulus of the various Th-Ti based alloys. The calculated bulk modulus for $\mathrm{Ti}$, Th and $\mathrm{ThTi}_{3}$ (in $\mathrm{Cr}_{3} \mathrm{Si}$ structure) are $118.48 \mathrm{GPa}, 58.21 \mathrm{GPa}$ and 100.98 GPa respectively. Other alloys of Th-Ti based systems have lower bulk modulus compared to $\mathrm{ThTi}_{3}$ (in $\mathrm{Cr}_{3} \mathrm{Si}$ structure). $\mathrm{ThTi}_{3}$ (in $\mathrm{Al}_{3} \mathrm{Ti}$ structure) has the largest magnitude of shear modulus and Young's modulus compared to the other alloys. The mean velocity calculated from shear (transverse) velocity and longitudinal velocity is used to determine the Debye temperature. The 
Debye temperature $\left(\theta_{D}\right)$ gives an indication of the stiffness of the material. $\operatorname{ThTi}_{3}\left(\mathrm{Al}_{3} \mathrm{Ti}\right), \mathrm{Ti}, \mathrm{ThTi}_{3}$ (in $\mathrm{Cr}_{3} \mathrm{Si}$ structure) has $\theta_{D}$ of $445.0 \mathrm{~K}, 426.1 \mathrm{~K}, 346.7 \mathrm{~K}$ respectively. This shows that the alloying of Th with Ti leads to considerable improvement in the mechanical properties of Th metal.

TABLE III. The bulk, shear and Young's moduli (Hill values in GPa), acoustic velocities $\left(m s^{-1}\right)$, and Debye temperature $(\mathrm{K})$

\begin{tabular}{rccccccc}
\hline \hline & $B_{H}$ & $G_{H}$ & $Y_{H}$ & $v_{s}$ & $v_{l}$ & $v_{m}$ & $\Theta_{D}$ \\
\hline $\mathrm{Th}$ & 58.21 & 24.94 & 64.72 & 1401 & 2684 & 1568 & 149.7 \\
$\mathrm{ThTi}_{3}\left(\mathrm{Cr}_{3} \mathrm{Si}\right)$ & 100.9 & 64.65 & 159.8 & 2798 & 4760 & 3101 & 346.7 \\
$\mathrm{ThTi}_{3}\left(\mathrm{Cu}_{3} \mathrm{Au}\right)$ & 92.70 & 31.38 & 84.58 & 1986 & 4112 & 2232 & 246.5 \\
$\operatorname{ThTi}_{3}\left(\mathrm{Al}{ }_{3} \mathrm{Ti}\right)$ & 89.80 & 108.7 & 207.7 & 3697 & 5432 & 4030 & 445.0 \\
$\operatorname{ThTi}_{(\mathrm{CsCl})}$ & 79.82 & 12.19 & 34.79 & 1106 & 3106 & 1257 & 131.0 \\
$\operatorname{ThTi}_{(\mathrm{CuAu})}$ & 77.45 & 24.81 & 67.16 & 1592 & 3360 & 1791 & 185.5 \\
$\operatorname{ThTi}(\mathrm{CuPt})$ & 73.19 & -52.86 & -430.0 & - & - & - & - \\
$\operatorname{ThTi}(\mathrm{NiAs})$ & -484.0 & 929.3 & 39630 & 15670 & 14130 & 15090 & 1138 \\
$\operatorname{Th}_{3} \mathrm{Ti}\left(\mathrm{Cr}{ }_{3} \mathrm{Si}\right)$ & 66.17 & 29.52 & 77.07 & 1626 & 3073 & 1817 & 178.9 \\
$\operatorname{Th}_{3} \mathrm{Ti}(\mathrm{Cu} 3 \mathrm{Au})$ & 69.55 & 30.37 & 79.41 & 1638 & 3118 & 1832 & 181.1 \\
$\operatorname{Th}_{3} \mathrm{Ti}\left(\mathrm{Al}{ }_{3} \mathrm{Ti}\right)$ & 64.84 & 31.24 & 80.63 & 1657 & 3060 & 1849 & 183.2 \\
$\mathrm{Ti}$ & 118.5 & 51.44 & 134.1 & 3257 & 6223 & 3643 & 426.1 \\
\hline \hline
\end{tabular}

\section{B. Phonon dispersion}

The phonon frequency of crystalline structures is one of the basic aspects when considering the phase stability, phase transformations, and the thermodynamics of these materials. The absence of imaginary (negative frequencies in the figures) frequencies in phonon dispersion curve and the phonon density of states give an indication that the structure is dynamically stable or vice versa. The phonon frequency for various Th-Ti based alloys in the NM phase using PBEsol is presented in Fig. 1. The use of PBE or PBEsol exchange correlation functional does not lead to a change in the lattice dynamics of these alloys. In Fig. 1a, $1 \mathrm{~b}$ and $1 \mathrm{c}$, the phonon plots of Th, Ti and $\mathrm{ThTi}_{3}$ (in $\mathrm{Cr}_{3} \mathrm{Si}$ structure) are shown respectively. They possess no imaginary phonon frequencies mode. Hence, they are structurally and thermodynamically stable. While, the other structures considered 
have various degree of instabilities due to the presence of imaginary frequencies. The phonon frequencies for the unstable structures are presented for completeness.

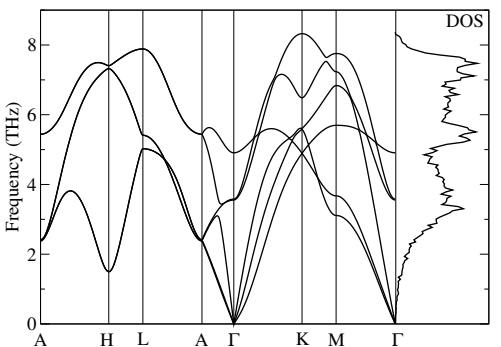

(a) Th in FCC

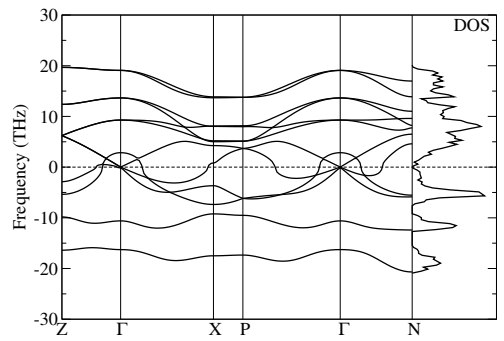

(d) $\mathrm{Th}_{3} \mathrm{Ti}$ in $\mathrm{Al}_{3} \mathrm{Ti}$

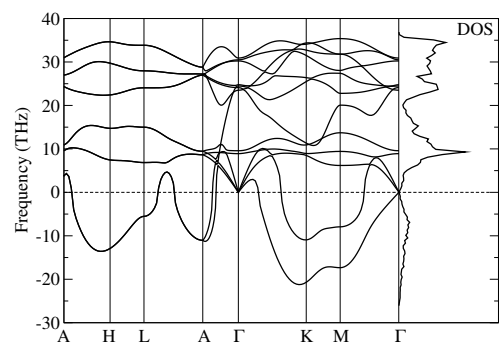

(g) ThTi in NiAs

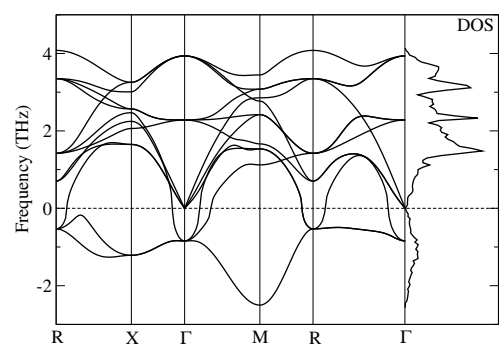

(j) $\mathrm{Th}_{3} \mathrm{Ti}$ in $\mathrm{Cu}_{3} \mathrm{Au}$

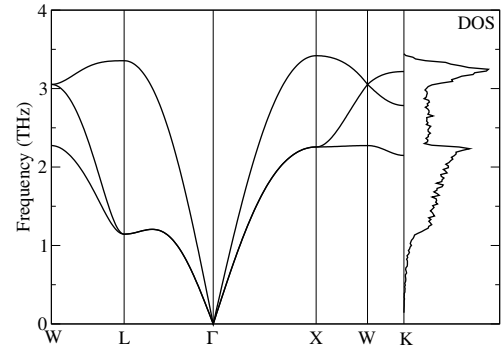

(b) $\mathrm{Ti}$ in $\mathrm{HCP}$

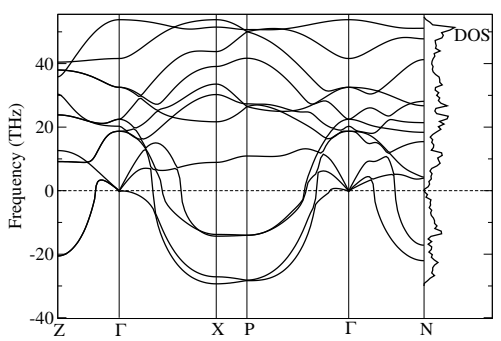

(e) $\mathrm{ThTi}_{3}$ in $\mathrm{Al}_{3} \mathrm{Ti}$

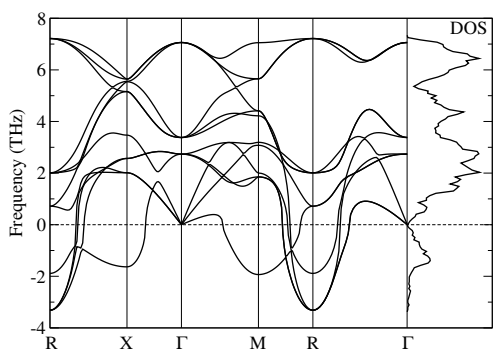

(h) $\mathrm{ThTi}_{3}$ in $\mathrm{Cu}_{3} \mathrm{Au}$

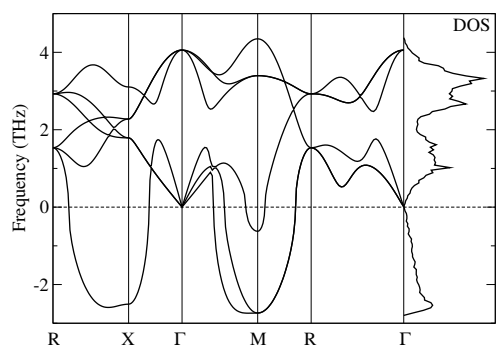

(k) ThTi in $\mathrm{CsCl}$

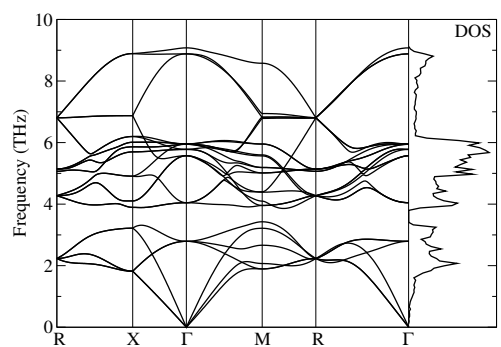

(c) $\mathrm{ThTi}_{3}$ in $\mathrm{Cr}_{3} \mathrm{Si}$

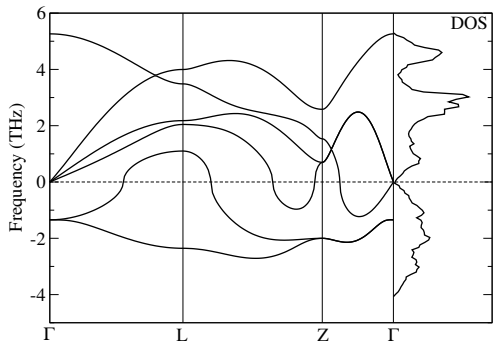

(f) ThTi in $\mathrm{CuPt}$

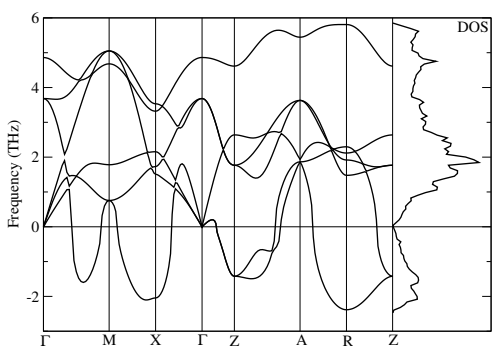

(i) ThTi in $\mathrm{CuAu}$

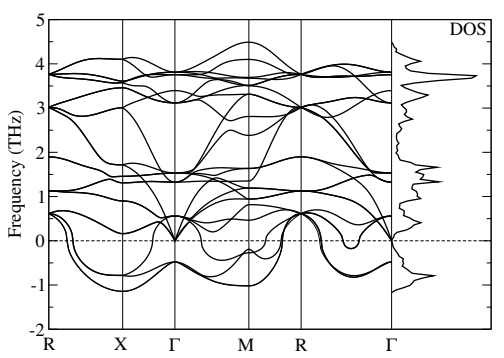

(1) $\mathrm{Th}_{3} \mathrm{Ti}$ in $\mathrm{Cr}_{3} \mathrm{Si}$

FIG. 1. Phonon dispersion and phonon density of state (DOS) plots for Th, Ti and Th-Ti based alloys in various ordered crystalline structures 
$\mathrm{ThTi}_{3}$ (in $\mathrm{Cu}_{3} \mathrm{Au}$ and in $\mathrm{Al}_{3} \mathrm{Ti}$ structures) have the same ratio of atoms as $\mathrm{ThTi}_{3}$ (in $\mathrm{Cr}_{3} \mathrm{Si}$ structure). It is interesting to note that $\mathrm{ThTi}_{3}$ (in $\mathrm{Cu}_{3} \mathrm{Au}$ and in $\mathrm{Al}_{3} \mathrm{Ti}$ structures) is stable at the $\Gamma$-point but possess few imaginary frequencies. This implies that the structure might transform to another structure or undergo pressure-induced stabilization. Therefore, these ordered structures with imaginary frequencies do not exist.

Isotropic pressure was applied to $\mathrm{ThTi}_{3}$ (in $\mathrm{Cu}_{3} \mathrm{Au}$ and in $\mathrm{Al}_{3} \mathrm{Ti}$ structures) with the aim of stabilizing or transforming these structures to its stable form. These structures did not undergo any structural stabilization or phase transformation with change in pressure. This can be attributed to the size of the unit cell and the actual number of atoms.

In the same line, isotropic pressure is applied to the dual alloys of $\operatorname{ThTi}_{3}$ (in $\mathrm{Cu}_{3} \mathrm{Au}$ and in $\mathrm{Al}_{3} \mathrm{Ti}$ structures) which is $\mathrm{Th}_{3} \mathrm{Ti}$ (in $\mathrm{Cu}_{3} \mathrm{Au}$ and in $\mathrm{Al}_{3} \mathrm{Ti}$ structures). The phonon dispersion is evaluated to determine its stability. Pressure induced stabilization of these structures occurred for $\mathrm{Th}_{3} \mathrm{Ti}$ (in $\mathrm{Cu}_{3} \mathrm{Au}$ and in $\mathrm{Al}_{3} \mathrm{Ti}$ structures) respectively. For $\mathrm{Th}_{3} \mathrm{Ti}$ alloy (in $\mathrm{Cu}_{3} \mathrm{Au}$ structure), a pressure of about $324.7 \mathrm{GPa}$ is applied to the dynamically unstable structure to achieve a stable

structure. Also $\mathrm{Th}_{3} \mathrm{Ti}$ alloy (in $\mathrm{Al}_{3} \mathrm{Ti}$ structure), a pressure of about $-3.574 \mathrm{GPa}$ was applied to the dynamically unstable structure to achieve a stable structure. The energies of formation of these pressure stabilized structures are positive and higher than that of $\mathrm{ThTi}_{3}$ alloy (in $\mathrm{Cr}_{3} \mathrm{Si}$ structure). Therefore, Th-Ti based alloys is most likely to be found as $\mathrm{ThTi}_{3}$ (in $\mathrm{Cr}_{3} \mathrm{Si}$ structure) rather than the pressure stabilized structures.

The phonon dispersion of $\mathrm{ThTi}_{3}$ alloy (in $\mathrm{Cr}_{3} \mathrm{Si}$ structure) has a splitting at $\Gamma$-point between the LO-TO, owing to the fact that $\mathrm{Th}$ atom is heavier than $\mathrm{Ti}$ atom. The low lying frequency modes are governed by the dynamics of heavy Th atoms, whereas the higher frequencies are governed by the dynamics of lighter $\mathrm{Ti}$ atoms.

\section{Heat of formation}

The Gibbs free energy of the various alloys is calculated. These calculations are performed at zero temperature and zero pressure, hence the Gibbs free energy reduces to the heat of formation, $\Delta \mathrm{H}$. The calculated heat of formation of the various alloys is investigated to determine its relative stability. Th and Ti metal are used as the reference systems to obtain the heat of formation for the alloys as described in the methodology. This is because they are the basic constituent of the alloy. The heat of formation for all the structures are presented in Table I for the NM phase with PBEsol 
pseudopotential. The structure with the lowest heat of formation for the investigated Th-Ti based alloys using the PBEsol (with NM) is ThTi (in $\mathrm{CsCl}$ structure). This structure is highly elastically and dynamically unstable.

TABLE IV. The lattice constant $\mathrm{a}_{0}(\AA)$, exchange-correlation functional, method, heat of formation (eV) for Th, Ti and $\mathrm{ThTi}_{3}\left(\mathrm{Cr}_{3} \mathrm{Si}\right)$ alloy using PBE and PBEsol. where non-magnetic, spin polarization and spin-orbit coupling is represented by NM, SP and SOC respectively

\begin{tabular}{lcccc}
\hline \hline & $a_{0}(\AA)$ & functional & method & $E_{f}(\mathrm{eV})$ \\
\hline $\mathrm{ThTi}_{3}\left(\mathrm{Cr}_{3} \mathrm{Si}\right)$ & 5.409 & PBE & SOC & 0.851 \\
$\mathrm{ThTi}_{3}\left(\mathrm{Cr}_{3} \mathrm{Si}\right)$ & 5.406 & PBE & $\mathrm{SP}$ & 1.003 \\
$\mathrm{ThTi}_{3}\left(\mathrm{Cr}_{3} \mathrm{Si}\right)$ & 5.406 & PBE & $\mathrm{NM}$ & 1.014 \\
$\mathrm{ThTi}_{3}\left(\mathrm{Cr}_{3} \mathrm{Si}\right)$ & 5.322 & PBEsol & SOC & 0.591 \\
$\operatorname{ThTi}_{3}\left(\mathrm{Cr}_{3} \mathrm{Si}\right)$ & 5.325 & PBEsol & SP & 0.727 \\
$\mathrm{ThTi}_{3}\left(\mathrm{Cr}_{3} \mathrm{Si}\right)$ & 5.325 & PBEsol & NM & 0.740 \\
\hline \hline
\end{tabular}

To accurately determine the total energy and the heat of formation for the stable alloys the inclusion of SOC is essential for these alloys. We calculated the heat of formation for $\mathrm{ThTi}_{3}$ alloy (in $\mathrm{Cr}_{3} \mathrm{Si}$ structure) using the PBEsol functional with $\mathrm{SOC}$ to be $0.591 \mathrm{eV}$ as listed in Table IV. Our calculated heat of formation for the system using PBEsol with SOC is less than NM and SP methods. In addition, using PBE functional with NM, SP and SOC method this results in a greater heat of formation compared to PBEsol with SOC. Generally, the inclusion of SOC leads to an effective lowering of the heat of formation in the structure for both GGA (PBE and PBEsol) functional.

The positive heat of formation of our system implies that the system will not occur spontaneously rather will require a positive energy input to create this alloy. The low value obtained for the heat of formation gives an indication that the formation of a metastable ordered alloy of Th and $\mathrm{Ti}$ is possible.

\section{THE PROPOSED METASTABLE ALLOY}

The band structure, density of states, valence charge density distribution and electron localization function $(\mathrm{ELF})^{25-27}$ for the metastable system under consideration are presented to analyze 
the electronic properties and bonding character.

The metastable alloy obtained from our investigation is $\mathrm{ThTi}_{3}$ alloy (in $\mathrm{Cr}_{3} \mathrm{Si}$ structure) as shown in Fig. 2. The alloy has the $P m-\overline{3} n$ space group (with group number 223 ). And the Th atoms in the unit cell are located in the $(0.0,0.0,0.0)$ and $(0.5,0.5,0.5)$ positions. The Ti atoms are located in the $(0.5,0.25,0)$ and $(0.5,0.75,0)$.

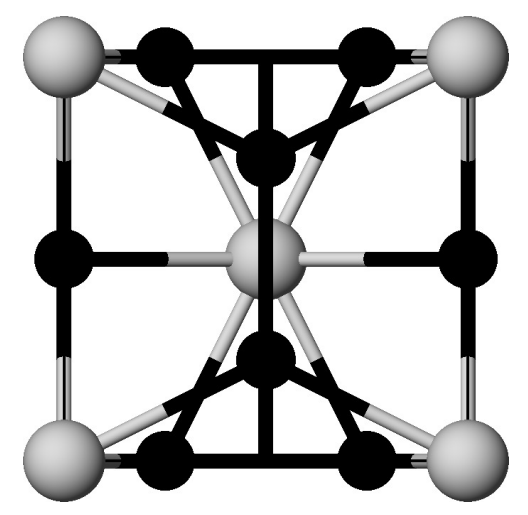

FIG. 2. The Structure of $\mathrm{ThTi}_{3}$ in $\mathrm{Cr}_{3} \mathrm{Si}$ where the white atoms represents $\mathrm{Th}$ atoms and the black atoms represents Ti atoms.

In Table IV, we present the GGA (PBE and PBEsol) results of the lattice parameter of the proposed metastable alloy. The difference in the lattice parameter obtained using PBE and PBEsol for the metastable system is about $1.6 \%$. It should be mentioned here that $\mathrm{ThTi}_{3}$ alloy (in $\mathrm{Cr}_{3} \mathrm{Si}$ structure) irrespective of the initial magnetic configuration gives a NM ground state as previously stated for all Th-Ti based alloys. This is corroborated by the insignificant difference in the lattice parameters between the NM, SP and SOC phase of the structure.

\section{A. Band structure and partial density of states}

In Fig. 3, we present the calculated band structures for Th, Ti and $\mathrm{ThTi}_{3}$ (in $\mathrm{Cr}_{3} \mathrm{Si}$ structure) along the high symmetry lines of BZ, and this evidently shows that they are all metallic structures. Th and Ti metals are comprised of two groups of bands with a huge difference of about $200 \%$ between the $\mathrm{Th}$ and $\mathrm{Ti}$ valence bands. This difference in the valence bands suggests difficulty in bonding between these two metals. It is no surprise then that only one plausible structure is obtained from our investigation.

The band structure of $\mathrm{ThTi}_{3}$ (in $\mathrm{Cr}_{3} \mathrm{Si}$ structure) consists of three isolated groups of valence bands with a slight energy dispersion $\mathrm{E}(\mathrm{k})$, which is typical of crystals with a relatively low degree 


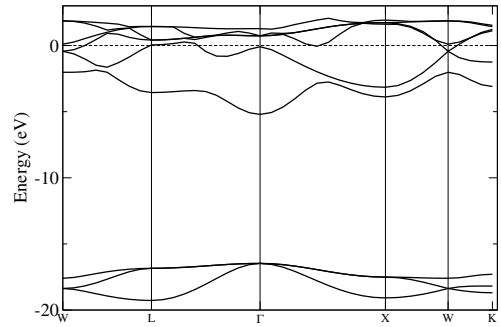

(a) Band Structure of Th

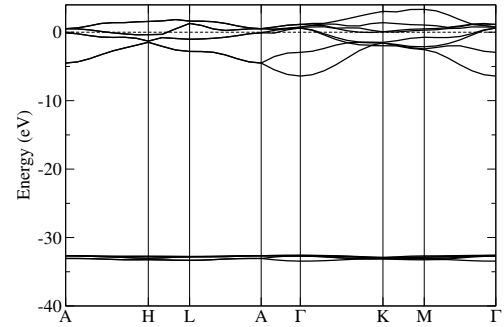

(b) Band Structure of Ti

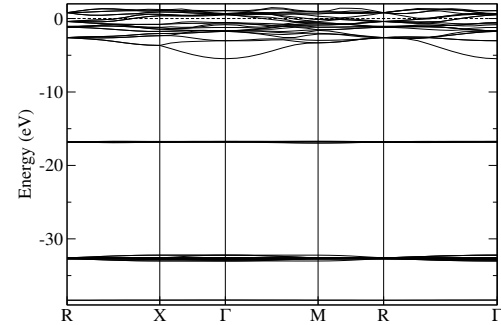

(c) Band Structure of $\mathrm{ThTi}_{3}$

FIG. 3. The band structure for $\mathrm{Th}$, $\mathrm{Ti}$ and $\mathrm{ThTi}_{3}$ alloy (in $\mathrm{Cr}_{3} \mathrm{Si}$ structure) with the Fermi energy level set (dashed line) at zero.

of orbital hybridization. ${ }^{28}$ The lowest group of band is from the Th $s$-orbital and is located around $-38 \mathrm{eV}$. The following bands in Fig. $3 \mathrm{c}$ are from the Th $p$-orbital and are located around $-21 \mathrm{eV}$ and $-15 \mathrm{eV}$ with a slight contribution from the Ti $p$-orbital in later bands. These bands have a quasi-core character. The broadest occupied band is in the range of $-5.5 \mathrm{eV}$ to $1.5 \mathrm{eV}$.

The PDOS for the metastable alloy is presented to highlight the effect of orbital hybridization around the Fermi level. The Th $(d$ and $f$ )-orbitals and the Ti ( $p$ and $d$ )-orbitals are presented in PDOS for $\mathrm{ThTi}_{3}$ (in $\mathrm{Cr}_{3} \mathrm{Si}$ structure) in Fig 4. The most dominant contribution at the Fermi level is from the Ti $d$-orbital while, the Th ( $d$ and $f$ )-orbitals provide almost equal contribution of states. The effect Ti $p$-orbital is marginal in determining the metallicity of $\mathrm{ThTi}_{3}$ (in $\mathrm{Cr}_{3} \mathrm{Si}$ structure). Looking at the contribution from Th, the dominant state in the conduction band is the Th $f$-orbital and in the valence band is the Th $d$-orbital. The system is very sensitive to the concentration of $\mathrm{Ti}$ atoms due to its overall contribution in determining the metallicity of the alloy. Hence, the configuration and concentration of $\mathrm{Ti}$ atoms will overall determine the electronic nature of this alloy.

\section{B. Valence charge density}

The computed valence charge density for $\mathrm{ThTi}_{3}$ alloy (in $\mathrm{Cr}_{3} \mathrm{Si}$ structure) is presented to elucidate the bonding character. Fig. 5 shows the computed charge density distribution plot for $\mathrm{ThTi}_{3}$ alloy (in $\mathrm{Cr}_{3} \mathrm{Si}$ structure) in the (100) plane. The valence charge density is indicated by the color scale on the side (blue is devoid of electrons and red has a high concentration of electrons). The valence charge densities plotted pertains to the valence states only summed over both spin direc- 


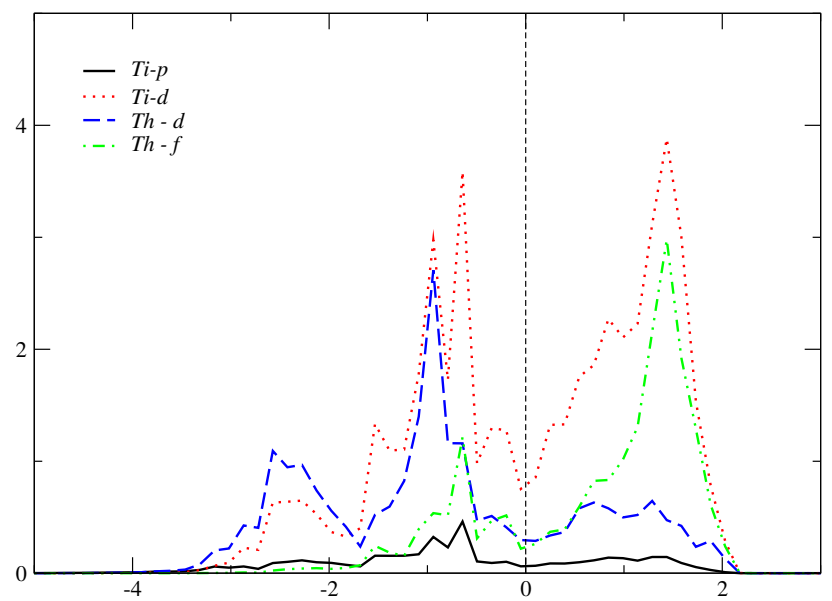

FIG. 4. (Color online) The partial density of states (PDOS) for $\mathrm{ThTi}_{3}$ (in $\mathrm{Cr}_{3} \mathrm{Si}$ structure). The DOS are calculated at the optimized geometry for the given density functional and magnetic ordering. The Fermi energy level is set at zero and is represented using dashed line.

tions.

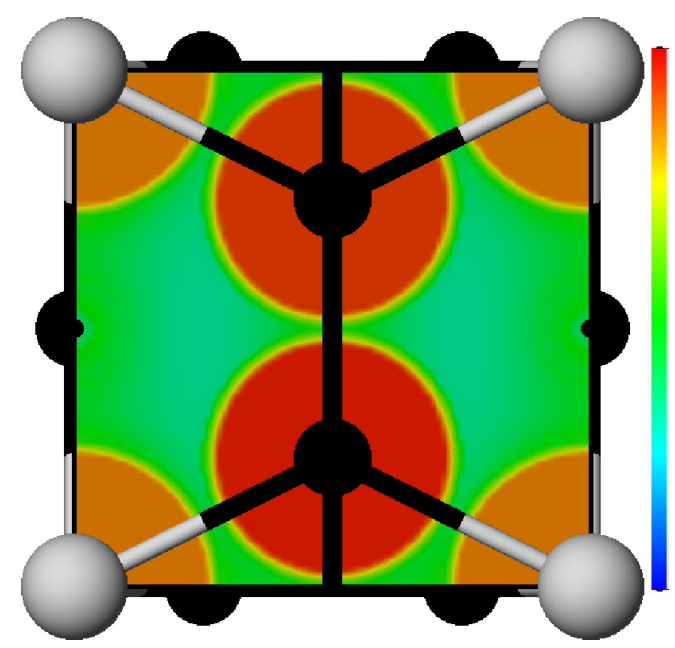

FIG. 5. (Color online) The valence charge density distribution for $\mathrm{ThTi}_{3}$ (in $\mathrm{Cr}_{3} \mathrm{Si}$ structure). The color pattern of the calculated valence charge density in the (001) plane, where the Th atom is depicted by the black balls and the Ti atoms by white balls. The color pattern of the valence charge density (scale: no electrons are represented as deep blue (0.0) and large concentration of electrons is represented as deep red (0.8); scale for remaining frames in increasing order of intensity: light blue, green, yellow, orange).

It is clearly seen that $\mathrm{ThTi}_{3}$ alloy (in $\mathrm{Cr}_{3} \mathrm{Si}$ structure) is metallic, characterized by a nearly spherical charge density distribution around the Th and Ti ions with very high concentration of 
valence charge and an appreciable charge density in all the interstitial regions. Hence, in k-space, appreciable band overlap exists between Th and Ti ions. Compared to the charge density in the rest of the interstitial region relatively high charge density bridges are present between the atomic spheres. This indicates ionization in the bridge sites suggestive of the presence of covalency.

In order to ascertain the presence of covalency in all of the bridge sites, we calculated the distribution of the ELF.

\section{Electron localization function for $\mathbf{T h T}_{3}$ (in $\mathrm{Cr}_{3} \mathrm{Si}$ structure)}

The ELF for the ground state bonding configuration of $\mathrm{ThTi}_{3}$ alloy in $\left(\mathrm{Cr}_{3} \mathrm{Si}\right.$ structure $)$ is evaluated to gain further insight into its bonding character and nature. ELF is given as $\left[1+\left(D / D_{h}\right)^{2}\right]^{-1}$, $D$ is $\tau-t_{W}$ represents an excess of the local kinetic energy due to the Pauli principle. Where $\tau=\frac{1}{2} \sum\left|\nabla \varphi_{i}\right|^{2}$ is the Kohn-Sham local kinetic energy, $\varphi_{i}$ are the Kohn-Sham orbitals, and $t_{W}$ is the value of $\tau$ in the absence of the Pauli principle $\left(\sim|\nabla \rho|^{2} / \rho\right.$ and $\rho$ being the charge density). $D_{h}$ being $D$ for the corresponding uniform electron gas $\left(\sim \rho^{5 / 3}\right)$. The spatial organization of ELF provides a basis for a proper classification of bonds ${ }^{27,29}$. Based on this definition ELF takes values ranging from 0 and 1 , where 1 represents perfect localization. The Th metal has more valence and core electrons than the Ti metal. They both exhibit similar features such as the large concentration of electrons around the atoms and an appreciable density of electrons away from the atoms (nondirectional), which is consistent with metallic bonding. In Fig. 6 the ELF is shown for the same plane as considered above for the valence charge-density distribution. Both picture are quite similar, with almost spherical shapes of the ELF around the Th atoms and a lower localization of the electrons on Ti atoms. Remarkably, the concentration of electrons between the Th and Ti bridges has a region of electron depletion compared to the $\mathrm{Ti}$ and $\mathrm{Ti}$ bridges. This observation gives an insight that the slight covalent character observed is from Ti atoms, which has a shared-electron pair. Hence, this particular crystal structure with more number of Ti atoms is stable compared to the corresponding structure $\left(\mathrm{Th}_{3} \mathrm{Ti}\right.$ alloy in $\left(\mathrm{Cr}_{3} \mathrm{Si}\right.$ structure $)$ with more $\mathrm{Th}$ atoms.

Based on these observations, the $\mathrm{ThTi}_{3}$ alloy in $\left(\mathrm{Cr}_{3} \mathrm{Si}\right.$ structure $)$ is predominantly metallic. 


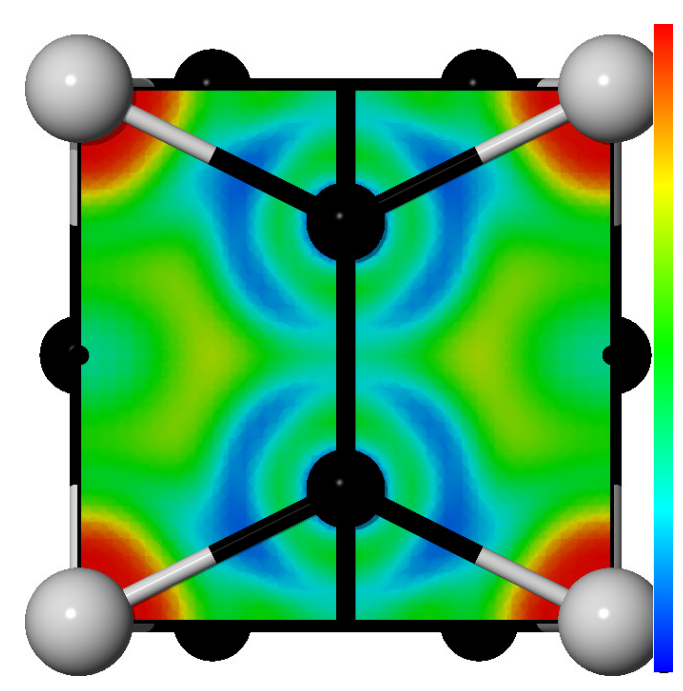

FIG. 6. (Color online) The electron localization function for $\mathrm{ThTi}_{3}$ (in $\mathrm{Cr}_{3} \mathrm{Si}$ structure). The color pattern of the calculated electron charge density in the (001) plane, where the Th atom is depicted by the black balls and the Ti atoms by white balls. The color pattern of the ELF (scale: no electrons are represented as deep blue (0.0) and large concentration of electrons is represented as deep red (0.1); scale for remaining frames in increasing order of intensity: light blue, green, yellow, orange).

\section{CONCLUSIONS}

This work is motivated by the usefulness of various alloys of Th and Ti, hence the search for the possibility of ordered alloys involving Th and Ti. We considered a number of different structures and stoichiometries involving these elements. A predicted metastable structure was established using arguments based on elastic constants, phonon frequencies, electron localization function, valence charge density, band structure, density of states and heat of formation.

The calculated phonon frequencies and elastic constants shows that $\mathrm{ThTi}_{3}$ (in $\mathrm{Cr}_{3} \mathrm{Si}$ structure) is dynamically stable at zero Kelvin and zero pressure as indicated by their positive values. This system using PBESol with the inclusion of SOC is energetically more favorable and gives an appropriate description with a heat of formation of $0.591 \mathrm{eV}$ compared with to the other methods. Though stabilization of some systems where achieved via pressure, the heat of formation of these systems are less favorable compared to $\mathrm{ThTi}_{3}$ (in $\mathrm{Cr}_{3} \mathrm{Si}$ structure). We propose $\mathrm{ThTi}_{3}$ (in $\mathrm{Cr}_{3} \mathrm{Si}$ structure) as a plausible metastable ordered alloy of Th-Ti based systems.

This should stimulate experimental efforts to synthesize this material. 


\section{ACKNOWLEDGMENTS}

We are grateful to the University of Pretoria for financial support. We thank Dr. Walter Meyer and Dr. Jannie Pretorius for assistance with the computational resources. Also we acknowledge Prof Richard Martin for assistance and the members of Theoretical and Computational Solid State Research Group at the University of Pretoria for useful discussions.

* Kingsley.Obodo@up.ac.za, 0027788489678

$\dagger$ Nithaya.Chetty@up.ac.za

1 I. Shein, K. Shein, and A. Ivanovskii, "First-principle study of B1-like thorium carbide, nitride and oxide," Journal of Nuclear Materials, vol. 353, pp. 19-26, July 2006.

2 I. Shein and A. Ivanovskii, "THORIUM COMPOUNDS WITH NON-METALS ELECTRONIC STRUCTURE, CHEMICAL BOND, AND PHYSICOCHEMICAL PROPERTIES,” Journal of Structural Chemistry, vol. 49, pp. 348-370, May 2008.

3 J. Adachi, K. Kurosaki, M. Uno, and S. Yamanaka, "A molecular dynamics study of thorium nitride," Journal of Alloys and Compounds, vol. 394, pp. 312-316, May 2005.

4 W. R. Evans, S. C. Barton, M. Clemens, and D. D. Allred, "Understanding DC-bias sputtered thorium oxide thin films useful in EUV optics," Proceedings of SPIE, vol. 5, no. 1, pp. 631711-631711-12, 2006.

5 T. Gouder, L. Havela, L. Black, F. Wastin, J. Rebizant, and B. P, "Synthesis and electronic properties of ThN films," Journal of Alloys and Compounds, vol. 336, pp. 73-76, Apr. 2002.

6 S. Mahmoud, "Characterization of thorium dioxide thin films prepared by the spray pyrolysis technique," Solid State Sciences, vol. 4, pp. 221-228, Feb. 2002.

7 H. Kleykamp, Gmelin handbook of inorganic and organometallic chemistry: Th, Thorium. Thorium carbides. Gmelin handbook of inorganic chemistry, Springer, Berlin, 1992.

8 R. Benz, A. Naoumidis, L. Gmelin, and D. Brown, Gmelin handbook of inorganic chemistry, 8th edition: Th: thorium. Compounds with nitrogen. No. no. 44, v. 3 in Gmelin handbook of inorganic chemistry, Springer-Verlag, 1987.

9 C. Keller, Gmelin Handbuch der Anorganischen Chemie, Thorium. Ternare and polynare Oxide des Thotiums. Gmelin Handbuch der Anorganischen Chemie, Springer, Berlin-Heidelberg-New York, 1976. 
10 D. Brown and H. Wedemayer, Gmelin handbook of inorganic and organometallic chemistry: Th, Thorium. Compounds with $\mathrm{S}, \mathrm{Se}$, Te and $\mathrm{B}$. Gmelin handbook of inorganic chemistry and Organometallic Chemistry, Springer, Berlin, 1993.

11 O. N. Carlson, J. M. Dickinson, H. E. Lunt, and H. A. Wilhem, "Thorium-Columbium and ThoriumTitanium Alloys Systems,” Transactions AIME, vol. 206, pp. 132-136, Feb. 1956.

12 E. T. Pedersen, M. Noack, and D. J. Verhoeven, "Eutectic alloys of thorium-nobium and thoriumtitanium," Journal of Materials Science, vol. 15, pp. 2115-2117, Sept. 1980.

13 P. Hohenberg and W. Kohn, "Inhomogeneous Electron Gas," Physical Review, vol. 136, no. 3B, p. 864, 1964.

14 G. Kresse and J. Furthmüller, "Efficient iterative schemes for ab initio total-energy calculations using a plane-wave basis set," Physical Review B, vol. 54, p. 11169, Oct. 1996.

15 J. P. Perdew, K. Burke, and M. Ernzerhof, “Generalized Gradient Approximation Made Simple.," Physical review letters, vol. 77, p. 3865, Oct. 1996.

16 J. P. Perdew, A. Ruzsinszky, G. I. Csonka, O. A. Vydrov, G. E. Scuseria, L. A. Constantin, X. Zhou, and K. Burke, "Restoring the Density-Gradient Expansion for Exchange in Solids and Surfaces," Physical Review Letters, vol. 100, p. 136406, Apr. 2008.

17 H. J. Monkhorst and J. D. Pack, "Special points for Brillouin-zone integrations," Physical Review B, vol. 13, no. 12, p. 5188, 1976.

18 M. Methfessel and A. T. Paxton, "High-precision sampling for Brillouin-zone integration in metals," Physical Review B, vol. 40, no. 6, p. 3616, 1989.

19 P. E. Blöchl, O. Jepsen, and O. K. Andersen, "Improved tetrahedron method for Brillouin-zine integrations," Physical Review B, vol. 49, no. 23, p. 16223, 1994.

20 Y. Le Page and P. Saxe, "Symmetry-general least-squares extraction of elastic data for strained materials from ab initio calculations of stress," Physical Review B, vol. 65, p. 104104, Feb. 2002.

21 O. L. Anderson, “A Smplified Method for Calculating the Debye Temperature from Elastic Constants,” Journal of Physics: Chemucak Solids, vol. 24, pp. 909-917, 1963.

22 Yong Lu, Da-Fang Li, Bao-Tian Wang, Rong-Wu Li, and Ping Zhang. Electronic structures, mechanical and thermodynamic properties of ThN from first-principles calculations. Journal of Nuclear Materials, 408:136-141, January 2011.

23 P. Soderlind, O. Eriksson, and B. Johansson, J. M. Wills "Electronic properties of $f$ metals using the generalized gradient approximation,” Physical Review B, vol. 50, no. 11, pp. 7291-7294, 1994. 
24 V. L. Moruzzi, J. F. Janak, and K. Schwarz, "Calculated thermal properties of metals," Physical Review $B$, vol. 37, no. 2, pp. 790-798, 1988 .

25 A. D. Becke and K. E. Edgecombe, "A simple measure of electron localization in atomic and molecular systems," The Journal of chemical physics, vol. 92, no. 9, pp. 5397-5403, 1990. Cited By (since 1996): 1408.

26 A. Savin, A. D. Becke, J. Flad, R. Nesper, H. Preuss, and H. G. von Schnering, “A New Look at Electron Localization," Angewandte Chemie International Edition in English, vol. 30, pp. 409-412, Apr. 1991.

27 B. Silvi and A. Savin, "Classification of chemical bonds based on topological analysis of electron localization functions," Nature, vol. 371, no. 6499, pp. 683-686, 1994. Cited By (since 1996): 998.

28 K. I. Shein, I. R. Shein, N. I. Medvedeva, V. G. Bamburov, and a. L. Ivanovskii, "Electronic structure of tetragonal thorium silicate in comparison with thorium dioxide," Doklady Physical Chemistry, vol. 409, pp. 198-201, July 2006.

29 N. V. Skorodumova, R. Ahuja, S. I. Simak, I. A. Abrikosov, B. Johansson, and B. I. Lundqvist, "Electronic, bonding, and optical properties of $\mathrm{CeO} 2$ and $\mathrm{Ce} 2 \mathrm{O} 3$ from first principles," Physical Review B, vol. 64, pp.115108, Aug. 2001. 